\title{
Analysis of Properties Hardware-Software System in Efficiency Index Under Uncertainty Component Structures
}

\author{
Murat B. Guzairov, Vladimir E. Gvozdev, Aliya S. Davlieva, Vyacheslav V. Teslenko ${ }^{1}$ \\ ${ }^{1}$ Department of Informatics and Robotics, Ufa State Aviation Technical University, \\ 12, K. Marx str. \\ Ufa, Russia \\ E-mail: aliyasr21@gmail.com
}

\begin{abstract}
The approach to the study of the components hardware-software complexes (HSC) survivability on the efficiency index for the interval uncertainty of the state's structure components is described. The problem of estimating the impact of HSC efficiency indexes variability in different operation modes on the evaluation under of target efficiency survivability is formulated. The study's results for various structures of HSC states are shown.
\end{abstract}

Keywords: Target efficiency, survivability, state structure, hardware-software complex.

\section{Introduction}

Information management support systems, which are essentially complex hardware and software systems, have now become a key factor in determining the safety and efficiency of complex systems $[1,2]$. The systemforming role of information support systems is due to the inseverable unity the complex tasks of managing complex objects implemented within the system approach and the tasks of constructing and using information support systems for solving control tasks [3, 4 , etc].

In the literature, various approaches and models of hardware-software complexes (HSCs) are described as a varieties complex systems, corresponding to different viewpoints on them; to different phases of the life cycle, etc. [5]. Within these approaches, the three-component architecture of information systems is discussed in one form or another: the hardware; information; algorithmic. Within these architectures, the importance of the functional safety is emphasized in different forms, irrespective of the purpose, scale, complexity of information systems.

The system characteristic "functional safety" can be associated with various static and dynamic characteristics, including vulnerability ([6, 7], etc.); survivability ([8-12], etc.); durability ([13], etc.). These characteristics are associated with different models constructed within various formal approaches.

Evolutionary development is characteristic for HSCs. Therefore, the inclusion of new components in the system requires a comprehensive analysis of both these components in the system and the study of changes in the HSC properties, as a result of a change in its structure.

Multicriteria analysis of alternatives to design solutions (in functional, information, organizational and other) in the early stages of the components life cycle included in the HSC, including studies of their survivability.

In this paper, the approach to the study of the components HSC survivability on the target efficiency index for the interval uncertainty of the state's structure components is described.

\section{Average Target Efficiency as an Index of the HSC Survivability}

Currently, literary sources [9, 10, etc.] give some different in form, but similar in content definitions of the concept "system survivability." The key points in these definitions are:

- The system's ability to adapt to unforeseen situations caused by changes in the environment or in the system structure;

- The system's ability to keep acceptable values consumer properties in this case.

In this paper, the HSC survivability is understood as the system's ability to adapt to the changed operating conditions, to resist negative effects by changing the properties of the structure components keeping acceptable system behavior for the consumer. 
Target efficiency [14] describes the conformity of the system functionality in terms of users to its intended purpose. Because of this, the target efficiency can be as an integral characteristic of the conformity the HSC properties to consumer's ideas about the "normal functioning of system".

\section{Task Statement}

The initial data of the task are:

a) The structure of the system states $S t r$ in the graph form $V=(S, \lambda)$, where $S_{i}$ - the nodes (states) of the system; $\lambda_{i j}$ - the edges connecting the $i$ and $j$ nodes, $i, j=\overline{1 ; n}$. A set $\{S\}_{1}^{n}$ is a complete group of incompatible events and each $i$ node is associated with an efficiency index $\ni_{i}$.

b) Known basic values of efficiency indexes $\Im_{i}^{(0)},(i=\overline{1 ; n})$, and the basic values deviations ranges $\left\{\Delta \Im_{i}^{(D)}, \Delta \Im_{i}^{(U)}\right\}$ of the efficiency indexes $\ni_{i}^{(0)}$ to the smaller and larger sides, i.e. $\exists_{i}^{(0)} \in\left[\Im_{i}^{(D)}, \ni_{i}^{(U)}\right]$, where

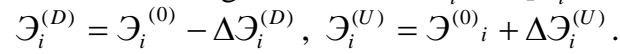

c) A well-known calculation functional based on $\Im_{i}^{(0)},(i=\overline{1 ; n})$ and $\lambda_{i j}$ basic integral characteristic of the target structure efficiency

$$
A:\left\{\ni_{i}^{(0)}, \lambda_{i j}\right\} \rightarrow \operatorname{Str}^{(0)} .
$$

d) The integral characteristic value of the target structure efficiency $S t r^{(\mathrm{k})}$, corresponding to the worsening of the efficiency index in the state $k$. The worsening is due to the change in the border set of the range $\Im_{\mathrm{k}}^{(P)} \in\left[\Delta \Im_{\mathrm{k}}^{(D)(P)}, \Delta \Im_{\mathrm{k}}^{(U)(P)}\right]$ to the worst side compared to $\ni_{\mathrm{k}}{ }^{(0)}$.

It is required: to what extent is it possible to compensate for the change in the integral characteristic of the target structure efficiency through a purposeful change in the border set of the range efficiency indexes for other than $k$ nodes, i.e.

$$
\Psi\left(\operatorname{Str}^{(0)}, \operatorname{Str}^{(k)}\right) \underset{\left\{\vartheta_{i}^{(D)}, \Im_{i}^{(U)}\right\}}{\longrightarrow} \min , i=\overline{1 ; n}, i \neq k .
$$

Here $\Psi(\bullet)-$ convolution rule.

\section{Estimating the Statistical Characteristic of Survivability}

Let there be a component of a distributed information system that can request memory / computing / information resources from other components of the system, at peak loads, or if it has free resources, to provide them on demand to other components that experience peak loads. Let the complete group of incompatible states be singled out $\mathrm{S}_{i},(i=\overline{1 ; n})$. The difference in states is described, for example, by the volumes of the resources requested or provided. At any given time, the system can only be in one of the states $S_{i}$ that is associated with an index of efficiency $\ni_{i} *$, characterizing the volume of resources provided or requested. If resources are requested, the index $\ni_{i}$ is negative; if provided - positive.

Let the target efficiency index of the system component is the index of the average volume of resources, determined by results of observations for a certain time period (statistical characteristic of the target efficiency):

$$
\ni_{\Sigma}=\sum_{i=1}^{n} \ni_{i} \cdot p_{i}
$$

where $p_{i}$ - the average relative time the system is in a state $\mathrm{S}_{i}$, and

$$
\sum_{i=1}^{n} p_{i}=1
$$

It is believed the closer $\ni_{\Sigma}$ to zero the system is functioning better, that is, during the observation period volume of resources provided to other components are equal to the volume of resources received from them. The transitions between states (states structure) defined by features of incoming requests from outside. Possible state structures are the "line", "star", "ring", "network". The number of nodes in each states structure is four. Transitions between states $i$ and $j$ is associated interval estimate of weighting characteristics $\lambda_{i j}$ (transitions intensity), and $\lambda_{i j} \in[0 ; 10]$ for $i, j=\overline{1 ; n}$.

In the maintenance of the HSC, due to many external and internal causes, deviations in the properties of structural components from the expected values are possible ( $\ni_{i}$ corresponds to interval uncertainty) $[16$, 17]. $\Im_{i}^{(0)}$ values are presented in Table 1 .

Table $1 . \ni_{i}^{(0)}$ values

\begin{tabular}{cccc}
\hline$\Im_{1}^{(0)}$ & $\ni_{2}{ }^{(0)}$ & $\Im_{3}{ }^{(0)}$ & $\Im_{4}{ }^{(0)}$ \\
\hline$[-10 ; 10]$ & {$[-10 ; 10]$} & {$[-10 ; 10]$} & {$[-10 ; 10]$}
\end{tabular}

It is required to compare the structures survivability by the index of the target efficiency in the case when the range $[-10 ; 0]$ is put in correspondence to $\ni_{1}{ }^{(P)}$.

Solution for the task

The basis solution for the task is the following computer experiment.

\footnotetext{
"If the $S_{i}$ is associated with a set of different types of resources, then $\mathcal{\exists}_{i}$ is a "generalized parameter", calculated on the basis of $\widetilde{Э}_{i j}: Э_{i}=\varphi\left(\widetilde{Э}_{i j}\right)$. Different formal schemes for determining the generalized parameter are described for example in [15]
} 
Step 1. The given number of times $Q$ were generated uniformly distributed random numbers corresponding to $\ni_{i}^{(0)(q)}, \lambda_{i j}^{(q)}$. For experiment, $Q$ was taken as 1000 .

Step 2. The resulting solutions the Kolmogorov equations [18] corresponding to the structures shown in Fig. 1, the final probabilities values of the states $p_{i}^{(q)}$ corresponding to $\lambda_{i j}^{(q)}$ were determined. Based on $p_{i}^{(q)}$ with $\ni_{i}^{(0)(q)}$ and (1) the values of the random variables ${\ni_{\Sigma}}^{(0)(q)}, q=\overline{1 ; Q}$ are determined.

Step 3. Based on the sample of independent random variables $\left\{\ni_{\Sigma}^{(0)}\right\}_{1}^{Q}$, the basic integral characteristics $S t r^{(0)}$ for each of the structures were calculated. As the basic integral characteristics were:

(a) Estimates of the integral distribution functions $\hat{F}^{(0)}\left(\ni_{\Sigma}\right)$.

(b) Estimates of mean value $\hat{M}\left(\Im_{\Sigma}^{(0)}\right)$ and standard deviation $\hat{\sigma}\left(\Im_{\Sigma}^{(0)}\right)$.

Step 4. The operations performed in steps 1-3 were repeated $Q$ times when the efficiency index corresponding to the $k$ system state $\ni_{k}$ changed in the range $Э_{k} \in[-10 ; 0]$. Based on the received samples of random numbers $\left\{\ni_{\Sigma}^{(P)}\right\}_{1}^{Q}$, the integral characteristics defined in step 3: $\hat{F}^{(P)}\left(Э_{\Sigma}\right) ; \hat{M}\left(\Im_{\Sigma}^{(P)}\right) ; \hat{\sigma}\left(\Im_{\Sigma}^{(P)}\right)$.

Fig. 1 shows the estimates of the integral distribution functions obtained as a result of the experiment.

The solid line in the figures shows the basic values of the integral indexes target efficiency. In Fig. 1 (a) $F^{(1,4)}\left(\ni_{\Sigma}\right)$ shows the case when indexes efficiency of the extreme structure nodes worsened, $F^{(2,3)}\left(\ni_{\Sigma}\right)$ shows the case when indexes efficiency of the second or third node worsened. In Fig. 1 (b) $F^{(1)}\left(Э_{\Sigma}\right)$ shows the case when the central node structure properties worsened; $F^{(2,3,4)}\left(\ni_{\Sigma}\right)$ - when the properties of one of the terminal nodes worsened.

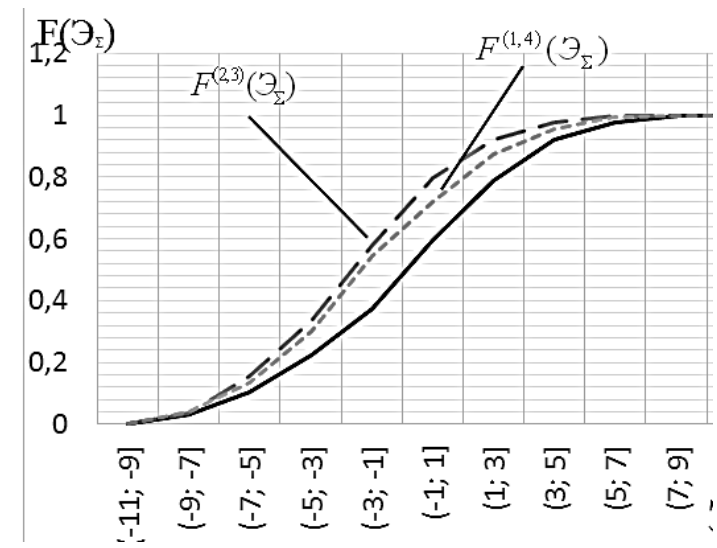

a) Structure "line"

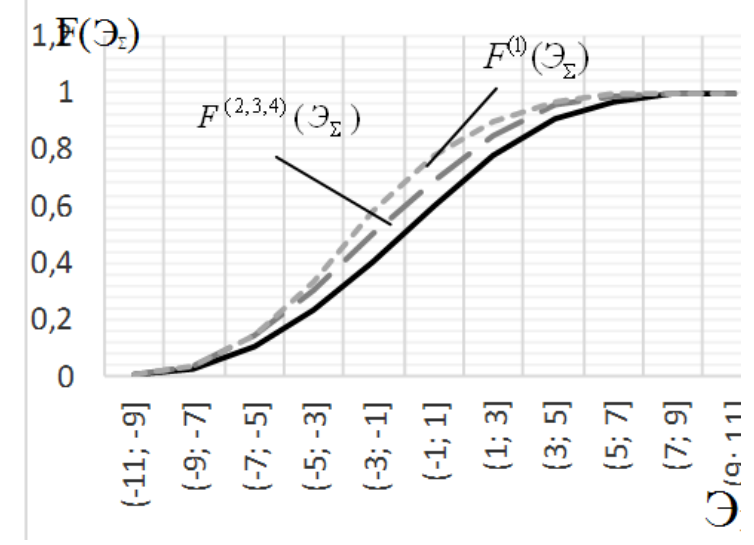

b) Structure "star"

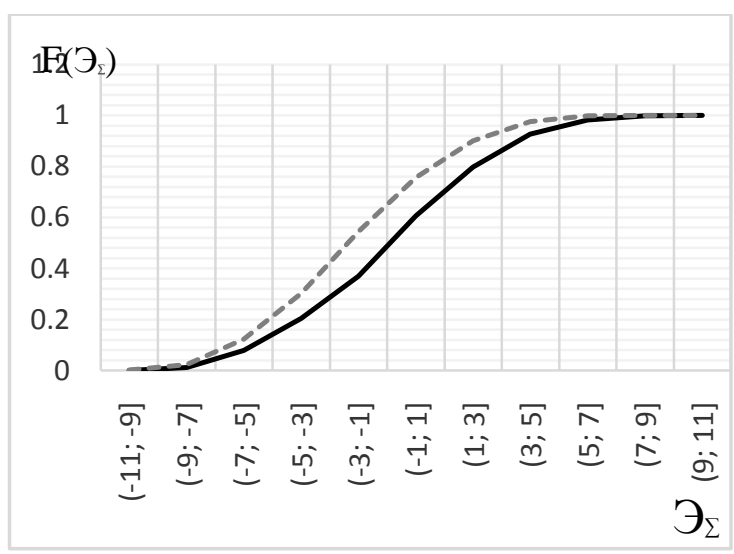

c) Structure "ring"

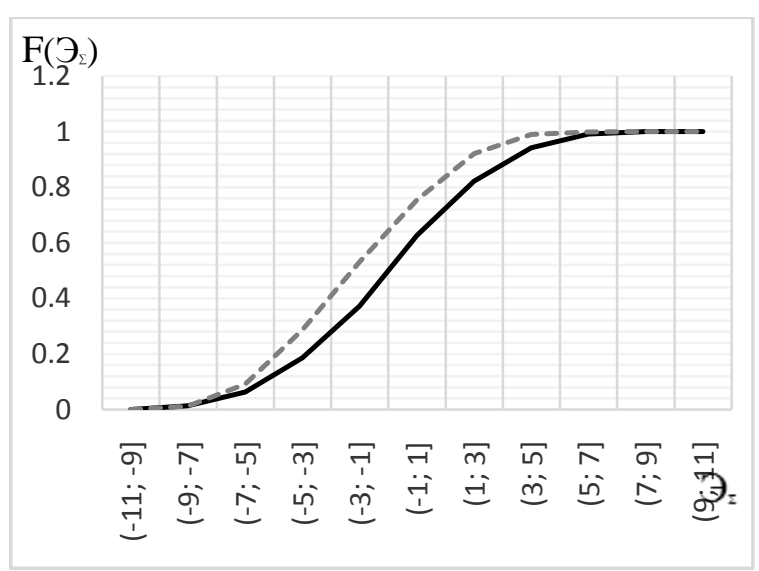

d) Structure "network"

Fig. 1. Estimates of the integral distribution functions for different structures. 


\section{Conclusion}

The results of the study allow us to conclude the linear structure was the most sensitive to the worsening of the indexes efficiency. If compensating the average target efficiency of terminal nodes state structure due to a purposeful change in the border set of the range for other nodes efficiency indexes, the average deviation is smaller but the spread is wider. For a linear structure, if the properties of terminal nodes worsen, the stability characteristic is higher than in the case of worsening in the nonterminal nodes characteristics. The results obtained can form the basis for comparing the alternatives between state transitions at the design stage of the HSC.

\section{Acknowledgements}

This work was supported by RFBR grant No. 17-0700351 "Methodological basics of dependability assurance of transmission systems telemetry information with use of intelligent data analysis technologies". HSC.

\section{References}

1. Lipaev V. V. Functional safety of software. Information bulletin "Jet Info", no. 8(135). (2004) 28 p. (in Russian).

2. Mostovoj A. Ja., Managing complex technical systems. designing software for remote sensing satellites: monograph (Moscow, Tehnosfera Publ., 2016) 352 p. (in Russian).

3. Apokin A. I. Informatics in the system of scientific knowledge of the XX and XXI centuries, in Proceedings "Cybernetics and Computer Science" to the 50th anniversary of the section of cybernetics of the M. Gorky House of Scientists RAS, 2006, pp. 21-35. (in Russian).

4. Jusupov R. M. Sokolov B. V. The problems of the cybernetics development and computer science at the present time, in Proceedings "Cybernetics and Computer Science" to the 50th anniversary of the section of cybernetics of the M. Gorky House of Scientists RAS, 2006, pp. 6-21. (in Russian).

5. Mostovoj A. Ja., Managing complex technical systems: designing software for remote sensing satellites: monograph, (Moscow, Tehnosfera Publ., 2016). 352 p. (in Russian).

6. Kubarev A. V., Approach to the formalization of the information systems vulnerabilities based on their classification characteristics, Cybersecurity issues, no. 2, (2013) 29-33. (in Russian).

7. Mahutov N. A., Reznikov D. O., Assessment of the technical systems vulnerability and its place in the procedure for risk analysis, Problems of risk analysis, Vol. 5, no. 3 (2008) 72-85. (in Russian).

8. Dedkov V. K., Vodolazskij V. I., Muhin A. V., Fesechko A. I., Survivability and safety for complex technical systems, Security issues and stability of systems theory, no. 4, (2002) 63-79. (in Russian).

9. Dodonov A. G., Landje D. V., Survivability of information systems (Kiev, Nauk. Dumka, 2011). 256 p. (in Russian).

10. Dodonov A. G., Flejtman D. V., Corporate Information Systems: Ensuring Vitality, Mathematical Machines and Systems, no. 4, (2005) 118-130. (in Russian).

11. Mahutov N. A., Reznikov D. O., Petrov V. P., Kuksova V. I., Evaluation of complex technical systems survivability, Problems of security and emergency situations, no. 3, (2009) 47-66. (in Russian).

12. Flejtman D. V., Solution of the ensuring problem the corporate information systems survivability with partial disruption of communication channels Registration, storage and processing of data, Vol. 7, no. 1, (2005) 5261. (in Russian).

13. Jones E. V., Lyford J. V., Qazi M. K., Solan N. J., Haimes Y.Y., Virginia's critical infrastructure protection study, in Systems and Information Engineering. Design Symposium (2004) pp. 177-182.

14. Lapigin U. N., Strategic management: a training manual (Moscow, Higher education, 2007). 174 p. (in Russian).

15. Gaskarov D. V., Golinkevich T. A., Mozgalevskij A. V., Technical state prediction and the reliability of electronic equipment, (Sovet. Radio, 1974) 222 p. (in Russian). 
16. Gvozdev V. E., Blinova D. V., Davlieva A. S., The analysis of functional suitability of hardware-software complexes based on apparatus of statistical indexes, Vestnik Samara State Technical University, Series "Technical sciences", no.2(54), (2017) 13-23. (in Russian).

17. Gvozdev V. E., Guzairov M. B., Blinova D. V., Davlieva A. S., Control of component alterations according with the target efficiency of data processing and control system, in Proceedings of the International conference "Information Technology and Nanotechnology". Session Data Science (Samara, Russia, 24-27 April, 2017) pp. 11-16.

18. Ventcel E. S., Operations research: tasks, principles, methodology, (Moscow, Nauka Publ., 1988) 208 p. (in Russian). 